\title{
Extend Convergence-Confinement Method for deep tunnels in poroelastic anisotropic medium
}

\author{
Nam-Hung Tran ${ }^{1, *}$, Thi-Thu-Nga Nguyen ${ }^{1}$, Duc-Phi Do $^{2}$, Tri-Ta Nguyen ${ }^{1}$ and Chu-Quang Cao ${ }^{1}$ \\ ${ }^{1}$ Le-Quy-Don Technical University, 236 Hoang-Quoc-Viet, Hanoi, Vietnam \\ ${ }^{2}$ University of Orléans, 8 Rue Léonard de Vinci, 45072 Orléans, France
}

\begin{abstract}
This paper is devoted to present a closed-form solution based on the approach of the conventional convergence-confinement method to study the effect of the movement of the tunnel face during the excavation on the stress-strain state of the surrounding medium and the interaction between the ground and the support for a deep tunnel in poroelastic anisotropic medium. In this study, a hydro-mechanical coupling behaviour of the ground at the steady-state of the groundwater flow will be taken into account. The obtained solution could be used as a quick tool to calibrate tunnels in elastic porous medium. Some numerical estimations indicate that, a hydro-mechanical model of the medium should be considered in tunnel design.
\end{abstract}

\section{Introduction}

One of the methods to study the tunnel excavation by two-dimensional plane strain problem, which can account for three-dimensional effect of the tunnel face to the sections behind and ahead of the face, is the convergence-confinement method (CCM). Following that, the effect of the movement of the tunnel face is then equivalent to the reduction of an inner fictive pressure on the tunnel wall. The CCM also takes into account the interaction between the ground mass and support and conditions of installation the support behind the tunnel face.

This approach is valid to calibrate the support/liner in the case of symmetric problem of deep, uniformly supported, circular tunnels embedded in an isotropic ground mass subjected to uniform in-situ stresses [1]. When the tunnels are placed in the poroelastic anisotropic medium, it is a challenge in studying the interaction of the ground-support. Extensions of the conventional CCM have been tried by some authors for the case of anisotropic initial pre-stress and elastic material [2-4]. However, a taking fully into account the anisotropic characterizes of the porous medium is still not mentioned in the literature.

In this paper, a closed-form solution based on the principle of the CCM to study the interaction between the ground and support for a deep tunnel in anisotropic poroelastic medium will be presented in which a hydro-mechanical coupling behaviour of the ground will be taken into account. It should be noted that we limit here by a steady state of

\footnotetext{
* Corresponding author: namhungcd@gmail.com
} 
groundwater flow. The solution in this work can be considered as an extension of the solution presented in [4] for the case of poroelastic anisotropic material.

\section{Interaction ground-support in anisotropic case}

Let us consider a deep tunnel with circular cross section of radius $R$ excavated in a transversely isotropic porous elastic medium saturated with an initial pore pressure $p_{f f}$ and total in-situ stresses $\sigma_{v}, \sigma_{h}, \tau_{v h}$ at infinity. The longitudinal axis of tunnel is parallel to the $z$ - axis in the Cartesian coordinates and the cross section lies on the vertical plane (x-y plane) which corresponds to the anisotropic plane of the medium. The porous medium is characterized by five mechanical parameters $E_{x}, E_{y}, v_{x z}, v_{y z}, G_{x y}$, two hydraulic conductivities $k_{x}$ and $k_{y}$, two coupled hydro-mechanical properties known as the Biot coefficients $b_{x}$ and $b_{y}$ [5].

The tunnel excavation is a successive process and the tunnel is prolonged in function of movement of tunnel face. At the instant of installation of the support $\left(t_{0}\right)$, the interest section is located at a certain distance from the face. Therefore, the tunnel wall had converged by an amount $(u d)$ before the installation of the support. Thus, the interactive problem of rock-support considering the tunnel face effect is an important issue not only to design the appropriate support but also to evaluate the work of rock mass before and after the installation of support.

It should be recalled that the conventional CCM is principally based on assumptions of the homogeneous, isotropic medium and isotropic in-situ stresses, i.e., hydrostatic pressure. Thus, the interactive problem degenerates to the one-dimensional problem on account of the axisymmetric conditions. However, this is not the case when the medium around the tunnel and/or the in-situ stresses are anisotropic. In this latter case, the behaviour of structures depends on the considered direction. The stresses applying on the extrados of the support include two components, the normal and shear stresses, which vary with respect to the studied position. Therefore, in this case the classical CCM is not applied directly. Fortunately, one could still address this problem based on the principle diagram of the CCM. This could be done through some extensions as detailed below.

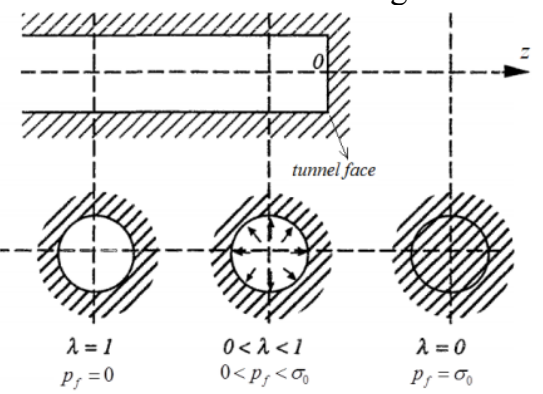

(a)

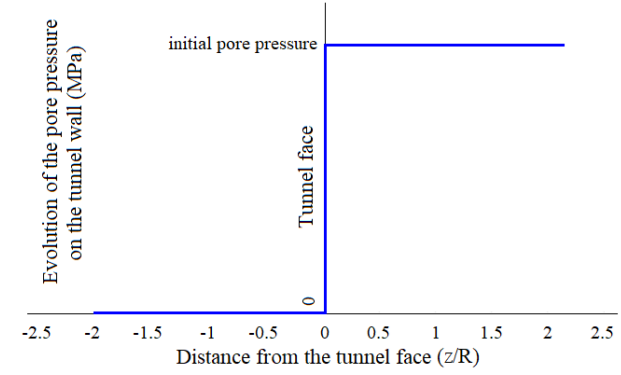

(b)

Fig.1. Evolution of the fictive pressure and pore pressure in function of distance from the study section to the tunnel face.

The 3D effect is also considered through the fictive pressure $\left(p_{f}\right)$ on the tunnel wall that decreases progressively over the excavation process. The evolution of this fictive pressure is characterized by the deconfinement rate $(\lambda)$ which depends on the distance $(z)$ between the considered section and the tunnel face [1].

In the framework of the poroelastic model of the ground, one assumes that if there is any change of pore pressure on the perimeter of tunnel, it is happening instantaneous when the tunnel face coincides with the studied section meaning that at the distance $z=0$. Fig. 1 illustrates the evolution law of the fictive pressure and pore pressure on the tunnel wall. 
The solution developed here considers only the condition of continuity at the interface between the rock mass and the support, i.e., perfect adhesion of rock mass - support.

In the CCM, it is necessary to establish the convergence law of the ground that shows the relationship between the convergence of the tunnel wall and the stresses imposed (convergence curve), and the response of the support described by a relationship between the stresses applying to its extrados and the corresponding displacement (confinement curve) as illustrated in Fig. $2[1,6]$. For the clarity purpose, keep in mind that the elastic behavior of surrounding rock mass is transversely isotropic while the behavior of the support is isotropic.

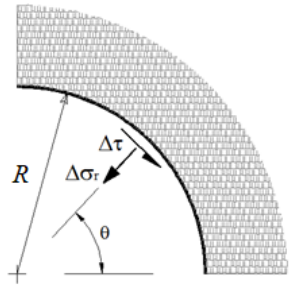

(a)

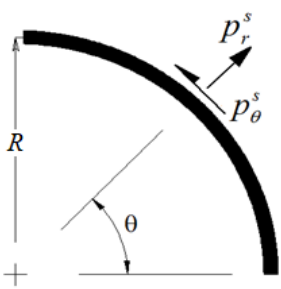

(b)

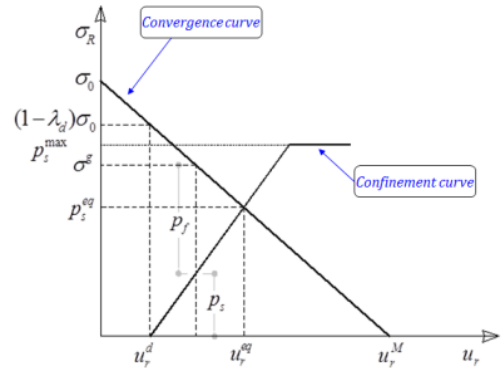

(c)

Fig.2. Stresses applying to the tunnel wall (a) and the extrados of the support after the support installation (b) and the convergence and confinement curves (c).

It should be noted that at each position on the ground-support interface, one has the diagram as presented in Fig.2c for the normal and shear stresses and for the radial and ortho-radial displacements separately. This means that, one cannot only rely on one diagram of the classical CCM to resolve the anisotropic problem as the isotropic one, but on the countless of diagram. From a practical point of view, this is unworkable. For all that, it is possible to address the problem through resolving the mathematic equations that based on the CCM principle presented below.

\subsection{Characteristic of the rock}

For the hydro-mechanical problem, the normal and shear stresses applying to the tunnel wall consist of two components, the first one is induced by mechanical phenomenon and the other is due to the hydraulic one. For the purpose of reducing the presentation, we will not present here these phenomena that the reader can see in chapter 2 of [5] and [7] for more detail.

Assuming the variation of the normal and shear stresses that applies on the tunnel wall are $\Delta \sigma_{r}, \Delta \tau$. Because of the symmetry of the geometry and the loading of the problem, the functions of these stresses exhibit the even and odd characteristic respectively. Thus, one can expand them in Fourier series forms as follows (see equations 2.20 and 2.53 in [5]):

$$
\begin{aligned}
& \Delta \sigma_{r}=\left(\sigma_{0}+\sigma_{0}^{p}\right)+\sum_{n=2,4,6}^{\infty}\left(\sigma_{n}^{a}+\sigma_{n}^{a, p}\right) \cos n \theta+\sum_{n=2,4,6}^{\infty}\left(\sigma_{n}^{b}+\sigma_{n}^{b, p}\right) \sin n \theta ; \\
& \Delta \tau=\sum_{n=2,4,6}^{\infty}\left(\tau_{n}^{a}+\tau_{n}^{a, p}\right) \sin n \theta+\sum_{n=2,4,6}^{\infty}\left(\tau_{n}^{b}+\tau_{n}^{b, p}\right) \cos n \theta .
\end{aligned}
$$

in which the coefficients $\sigma_{0}, \sigma_{n}^{a}, \sigma_{n}^{b}, \tau_{n}^{a}, \tau_{n}^{b}$ are related to the mechanical problem and the coefficients $\sigma_{0}^{p}, \sigma_{n}^{a, p}, \sigma_{n}^{b, p}, \tau_{n}^{a, p}, \tau_{n}^{b, p}$ are related to hydraulic one..

Similarly, the displacements on the tunnel wall are written in the form (see [4]): 


$$
\begin{aligned}
& \frac{u_{r}}{R}=\left(a_{0}+a_{0}^{p}\right)+\sum_{n=2,4,6}^{\infty}\left(a_{n}^{a}+a_{n}^{a, p}\right) \cos n \theta+\sum_{n=2,4,6}^{\infty}\left(a_{n}^{b}+a_{n}^{b, p}\right) \sin n \theta, \\
& \frac{u_{\theta}}{R}=\left(b_{0}+b_{0}^{p}\right)+\sum_{n=2,4,6}^{\infty}\left(b_{n}^{a}+b_{n}^{a, p}\right) \sin n \theta+\sum_{n=2,4,6}^{\infty}\left(b_{n}^{b}+b_{n}^{b, p}\right) \cos n \theta
\end{aligned}
$$

where $a_{0}, a_{n}^{a}, a_{n}^{b}, b_{0}, b_{n}^{a}, b_{n}^{b}$ and $a_{0}^{p}, a_{n}^{a, p}, a_{n}^{b, p}, b_{0}^{p}, b_{n}^{a, p}, b_{n}^{b, p}$ are coefficients relating to the mechanical and hydraulic phenomena respectively.

Noting that, the displacements in Eq.(2) are deduced from the stresses in Eq.(1) by applying the complex variable approach of Lekhnitskii [5]. Thus, the coefficients in Eq.(2) depend on the coefficients in Eq.(1) (see more detail in chapter 2 of [5]). The coefficients of Eq.(1), and thereby the ones of Eq.(2), can be determined by imposing the compatibility condition of displacements at the rock mass-support interface [5]. The expression of this compatibility condition of displacements will be present in section 2.3 of this paper.

From a practical point of view, it is not necessary to solve for all the terms of Eq.(1) and Eq.(2), as the contributions from the higher terms will be negligible. By truncating the series expansion to the order of $m$, the relationship between the displacement and the variation of stress on the tunnel wall is written in matrix form [4]:

$$
\frac{1}{R}\left(\begin{array}{l}
\mathbf{u}_{r} \\
\mathbf{u}_{\theta}
\end{array}\right)=\mathbf{G}\left(\begin{array}{l}
\Delta \boldsymbol{\sigma}_{r} \\
\Delta \boldsymbol{\tau}
\end{array}\right)
$$

in which:

$$
\begin{gathered}
\mathbf{u}_{r}=\left\{a_{0}+a_{0}^{p}, a_{1}^{a}+a_{1}^{a, p}, a_{2}^{a}+a_{2}^{a, p} \ldots, a_{m}^{a}+a_{m}^{a, p}, a_{1}^{b}+a_{1}^{b, p}, a_{2}^{b}+a_{2}^{b, p}, \ldots, a_{m}^{b}+a_{m}^{b, p}\right\}^{T} ; \\
\mathbf{u}_{\theta}=\left\{b_{1}^{a}+b_{1}^{a, p}, b_{2}^{a}+b_{2}^{a, p} \ldots, b_{m}^{a}+b_{m}^{a, p}, b_{1}^{b}+b_{1}^{b, p}, b_{2}^{b}+b_{2}^{b, p}, \ldots, b_{m}^{b}+b_{m}^{b, p}\right\}^{T} \\
\Delta \boldsymbol{\sigma}_{r}=\left\{\sigma_{0}+\sigma_{0}^{p}, \sigma_{1}^{a}+\sigma_{1}^{a, p}, \sigma_{2}^{a}+\sigma_{2}^{a, p}, \ldots, \sigma_{m}^{a}+\sigma_{m}^{a, p}, \sigma_{1}^{b}+\sigma_{1}^{b, p}, \sigma_{2}^{b}+\sigma_{2}^{b, p}, \ldots, \sigma_{m}^{b}+\sigma_{m}^{b, p}\right\}^{T} \\
\boldsymbol{\Delta} \boldsymbol{\tau}=\left\{\tau_{0}+\tau_{0}^{p}, \tau_{1}^{a}+\tau_{1}^{a, p}, \tau_{2}^{a}+\tau_{2}^{a, p}, \ldots, \tau_{m}^{a}+\tau_{m}^{a, p}, \tau_{1}^{b}+\tau_{1}^{b, p}, \tau_{2}^{b}+\tau_{2}^{b, p}, \ldots, \tau_{m}^{b}+\tau_{m}^{b, p}\right\}^{T}
\end{gathered}
$$

and $\mathbf{G}$ is the square matrix of order of $4 m+1$ which characterizes the behaviour of the ground. The relationship in Eq. (3) describes the convergence curve.

\subsection{Characteristic of the support}

The support is constituted by a circular annual of extrados radius $\mathrm{R}$ and of small thickness $t s$. One assumes that the support material is characterized by a linear isotropic elastic model whose parameters are the Young modulus $E_{s}$ and the Poisson coefficient $v_{s}$. The relationship between the stresses that apply to the extrados and the displacement of the support is written [8]:

$$
K_{f}\left(\frac{1}{R} \frac{\partial^{4} u_{r}}{\partial \theta^{4}}+\frac{2}{R} \frac{d^{2} u_{r}}{d \theta^{2}}+\frac{u_{r}}{R}\right)+K_{n}\left(\frac{u_{r}}{R}+\frac{1}{R} \frac{d u_{\theta}}{d \theta}\right)=p_{r}^{s},-K_{n}\left(\frac{1}{R} \frac{d u_{r}}{d \theta}+\frac{1}{R} \frac{\partial^{2} u_{\theta}}{\partial \theta^{2}}\right)=p_{\theta}^{s}
$$

where $p_{r}^{s}$ and $p_{\theta}^{s}$ are respectively the radial stress and shear stress applied to the extrados of the support; $u_{r}^{s}$ and $u_{\theta}^{s}$ are the radial displacement and ortho-radial displacement of the support; $K_{n}$ and $K_{f}$ are the normal stiffness and flexible stiffness moduli and they are given by the following expressions:

$$
K_{n}=\frac{E_{s}}{1-v_{s}^{2}} \frac{t_{s}}{R} ; \quad K_{f}=\frac{E_{s}}{1-v_{s}^{2}} \frac{I}{R} ; \quad I=\frac{t_{s}^{3}}{12}
$$

In the same way for the stresses acting on the tunnel wall, one has the Fourier expansions of the stresses imposing on the extrados of the support as follows [4]: 


$$
p_{r}^{s}=p_{0}+\sum_{n=2,4,6}^{\infty} p_{n}^{a} \cos n \theta+\sum_{n=2,4,6}^{\infty} p_{n}^{b} \sin n \theta, p_{\theta}^{s}=\sum_{n=2,4,6}^{\infty} q_{n}^{a} \sin n \theta+\sum_{n=2,4,6}^{\infty} q_{n}^{b} \cos n \theta
$$

and with the same form, we have the following relations for the displacements:

$$
\frac{u_{r}^{s}}{R}=c_{0}+\sum_{n=2,4,6}^{\infty} c_{n}^{a} \cos n \theta+\sum_{n=2,4,6}^{\infty} c_{n}^{b} \sin n \theta, \frac{u_{\theta}^{s}}{R}=d_{0}+\sum_{n=2,4,6}^{\infty} d_{n}^{a} \sin n \theta+\sum_{n=2,4,6}^{\infty} d_{n}^{b} \cos n \theta
$$

where $p_{n}^{a}, p_{n}^{b}, q_{n}^{a}, q_{n}^{b}$ and $c_{0}, c_{n}^{a}, c_{n}^{b}, d_{0}, d_{n}^{a}, d_{n}^{b}$ are the coefficients of the series. From Eqs. (5), (7) and (8), it is seen that the coefficients of Eq. (8) depend on those of Eq.(7). These coefficients are determined by the compatibility of stresses and displacement at the massifsupport interface (see expressions in [5]).

The relationship between the displacements and the stresses on the support can be also written in the matrix form as:

in which

$$
\frac{1}{R}\left(\begin{array}{c}
\mathbf{u}_{r}^{s} \\
\mathbf{u}_{\theta}^{s}
\end{array}\right)=\mathbf{K}^{s}\left(\begin{array}{c}
\mathbf{p}_{r}^{s} \\
\mathbf{p}_{\theta}^{s}
\end{array}\right)
$$

$$
\begin{array}{ll}
\mathbf{u}_{r}^{s}=\left\{c_{0}, c_{1}^{a}, c_{2}^{a} \ldots c_{m}^{a}, c_{1}^{b}, c_{2}^{b}, \ldots, c_{m}^{b}\right\}^{T} ; & \mathbf{u}_{\theta}^{s}=\left\{d_{1}^{a}, d_{2}^{a} \ldots d_{m}^{a}, d_{1}^{b}, d_{2}^{b}, \ldots, d_{m}^{b}\right\}^{T} \\
\mathbf{p}_{r}^{s}=\left\{p_{0}, p_{1}^{a}, p_{2}^{a} \ldots, p_{m}^{a}, p_{1}^{b}, p_{2}^{b}, \ldots, p_{m}^{b}\right\}^{T} ; & \mathbf{p}_{\theta}^{s}=\left\{q_{0}, q_{1}^{a}, q_{2}^{a} \ldots, q_{m}^{a}, q_{1}^{b}, q_{2}^{b}, \ldots, q_{m}^{b}\right\}^{T}
\end{array}
$$

and $\mathbf{K}^{s}$ is the square matrix of order of $4 m+1$ which characterizes the behaviour of the support. The relationship in Eq. (9) describes the confinement curve.

\subsection{Analytical solution for ground-support interaction}

As presented, in the convergence-confinement method, the influence of tunnel face on the considered section is taken into account by the inner fictive pressure $p^{f}$ whose evolution is governed by the deconfinement rate $\lambda(z)$. In the case of anisotropic in-situ stresses state, it is straightforward to determine the normal and shear initial stresses at the tunnel wall as below [5]:

$$
\sigma_{r 0}=\frac{1}{2}\left(\sigma_{v}-\sigma_{h}\right) \cos 2 \theta-\frac{1}{2}\left(\sigma_{h}+\sigma_{v}\right)-\tau_{v h} \sin 2 \theta, \quad \tau_{0}=\frac{1}{2}\left(\sigma_{h}-\sigma_{v}\right) \sin 2 \theta-\tau_{v h} \cos 2 \theta
$$

Thus, the normal and shear fictive pressure applying to the perimeter of the tunnel wall are determined by the relationships:

$$
p_{r}^{f}=(1-\lambda)\left[\frac{1}{2}\left(\sigma_{v}-\sigma_{h}\right) \cos 2 \theta-\frac{1}{2}\left(\sigma_{h}+\sigma_{v}\right)-\tau_{v h} \sin 2 \theta\right], p_{\theta}^{f}=(1-\lambda)\left[\frac{1}{2}\left(\sigma_{h}-\sigma_{v}\right) \sin 2 \theta-\tau_{v h} \cos 2 \theta\right]
$$

where $\sigma_{h}, \sigma_{v}, \tau_{v h}$ are the far-field initial stresses (Fig.1). It assumes that, the deconfinement rate is $\lambda_{d}=\lambda(d)$, with $d$ the distance from the tunnel face to the section of support installation, so one has stress variations on tunnel wall as follows:

$$
\Delta \sigma_{r}^{d}=\lambda_{d}\left[\frac{1}{2}\left(\sigma_{v}-\sigma_{h}\right) \cos 2 \theta-\frac{1}{2}\left(\sigma_{h}+\sigma_{v}\right)-\tau_{v h} \sin 2 \theta\right], \Delta \tau_{\theta}^{d}=\lambda_{d}\left[\frac{1}{2}\left(\sigma_{h}-\sigma_{v}\right) \sin 2 \theta-\tau_{v h} \cos 2 \theta\right]
$$

Therefore, the corresponding displacements of the tunnel wall at this stage are determined by following relationship:

$$
\frac{1}{R}\left(\begin{array}{c}
\mathbf{u}_{r}^{d} \\
\mathbf{u}_{\theta}^{d}
\end{array}\right)=\mathbf{G}\left(\begin{array}{c}
\Delta \boldsymbol{\sigma}_{r}^{d} \\
\Delta \boldsymbol{\tau}^{d}
\end{array}\right)=\lambda_{d} \mathbf{G}\left(\begin{array}{c}
\boldsymbol{\sigma}_{r}^{0} \\
\boldsymbol{\tau}^{0}
\end{array}\right)
$$


After installation of the support, the relationship between the stresses applying to the tunnel wall $\boldsymbol{\sigma}_{r}^{g}, \boldsymbol{\tau}^{g}$ and the stresses applying to the extrados of the support is written (see Fig.2c):

$$
\left(\begin{array}{c}
\boldsymbol{\sigma}_{r}^{g} \\
\boldsymbol{\tau}^{g}
\end{array}\right)=\left(\begin{array}{c}
\mathbf{p}_{r}^{s} \\
\mathbf{p}_{\theta}^{s}
\end{array}\right)+\left(\begin{array}{c}
\mathbf{p}_{r}^{f} \\
\mathbf{p}_{\theta}^{f}
\end{array}\right)
$$

The final equilibrium state reaches when $\mathbf{p}^{f}=0$, and hence (see Fig.2c):

$$
\left(\begin{array}{c}
\Delta \boldsymbol{\sigma}_{r}^{e q} \\
\Delta \boldsymbol{\tau}^{e q}
\end{array}\right)=\left(\begin{array}{c}
\boldsymbol{\sigma}_{r}^{0} \\
\boldsymbol{\tau}^{0}
\end{array}\right)-\left(\begin{array}{c}
\mathbf{p}_{r}^{s, e q} \\
\mathbf{p}_{\theta}^{s, e q}
\end{array}\right)
$$

The perfect contact condition implies the continuity of the radial and the tangential displacements between the ground and the support, so one has the equation:

$$
\mathbf{u}_{r}^{e q}-\mathbf{u}_{r}^{d}=\mathbf{u}_{r}^{s, e q}, \quad \mathbf{u}_{\theta}^{e q}-\mathbf{u}_{\theta}^{d}=\mathbf{u}_{\theta}^{s, e q}
$$

Hence, one can infer the stresses applying on the extrados of the support as follows:

$$
\left(\begin{array}{l}
\mathbf{p}_{r}^{s, e q} \\
\mathbf{p}_{\theta}^{s, e q}
\end{array}\right)=\left(1-\lambda_{d}\right)\left(\mathbf{G}+\mathbf{K}^{s}\right)^{-1}\left(\begin{array}{c}
\boldsymbol{\sigma}_{r}^{0} \\
\boldsymbol{\tau}^{0}
\end{array}\right)
$$

The equation (18) indicates that, if one knows the deconfinement rate of support installation $\lambda_{d}$, one can compute the stresses applying to the extrados of the support at the final equilibrium state of the ground - support from the initial stresses at the tunnel wall. And hence, the stress variations on the tunnel wall are deduced form Eq.(16). Thereafter, the displacements, and thereby the stress - strain state, of the ground as well as of the support are determined by the Eqs. (3) and (9).

It should be noted that, we applied successfully the complex variable approach of Lekhnitskii for the anisotropic hydro-mechanical problem in the case of deconfinement rate $\lambda_{d}=0$, i.e., the tunnel excavation and support installation occur simultaneously, in chapter 2 of [5] that is the special case of the solution presented in this paper.

\section{Application of the closed-form solution}

In this part, one considers a specific example of the deep tunnel of radius $R=2.6 \mathrm{~m}$ in anisotropic poro-elastic medium with the special attention on the interaction of rocksupport. The hydro-mechanical properties of the Callovo-Oxfordian clay-stone at depth of $500 \mathrm{~m}$ are chosen as follows [9]: $E_{x}=5600 \mathrm{MPa}, E_{y}=4000 \mathrm{MPa}, v_{x z}=0.3, v_{y x}=0.142$, $G_{x y}=1600 M P a, k_{x}=4 \times 10^{-13} \mathrm{~m} / \mathrm{s}, k_{y}=1.33 \times 10^{-13} \mathrm{~m} / \mathrm{s}, b_{x}=b_{y}=0.6$. Concerning the liner (with thickness $t_{s}=0.18 \mathrm{~m}$ ), the mechanical properties are chosen $E_{s}=20 \mathrm{GPa}$ and $v_{s}=0.3$. At the first stage, we validate the closed-form solution by comparing with one obtained from the numerical simulation based on the finite element method (FEM). One 2D model is built in the FEM code (Code_Aster) using the plane strain elements with 8 nodes. 


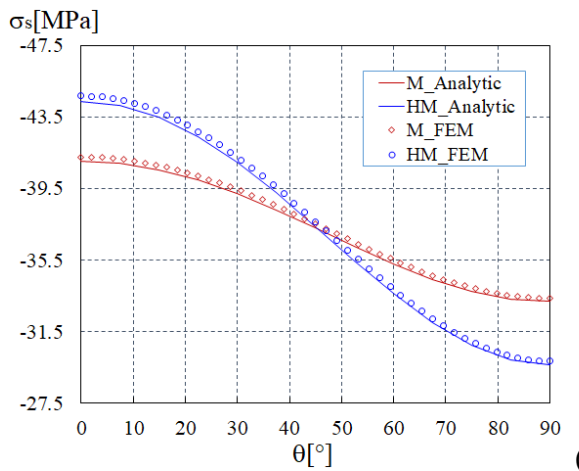

(a)

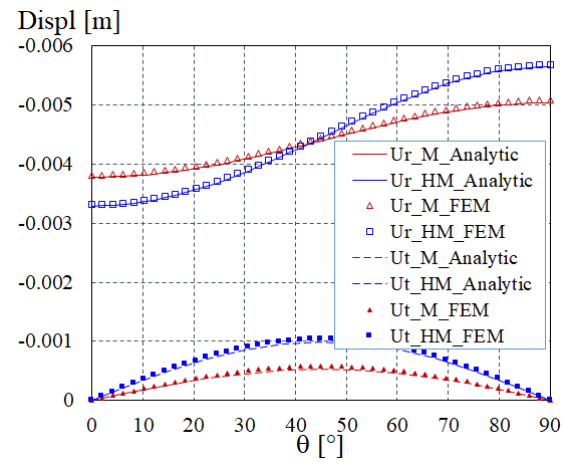

Fig.3. Interior fiber tangential stresses $\sigma_{s}(a)$ and radial and tangential displacements $U_{r}, U_{t}(b)$ of the liner in the case of $\lambda_{\mathrm{d}}=0.4$ in comparison between the analytical (Analytic) and numerical (FEM) solutions for the cases of mechanical problem (denoted by $\mathrm{M}$ ) and hydro-mechanical problem at steady state (denoted by HM).

It can be seen in Fig. 3 that, the closed-form solution give one very good agreement with the numerical solution. Fig. 3 shows also that, as long as the same total in-situ stresses, the stresses and the displacements of the liner in the hydro-mechanical case (saturated ground) are always higher than those of mechanical case (dry rock) due to the presence of seepage force induced by the anisotropic fluid flow.

At the second stage, one investigates now the influence of deconfinement rate $\lambda_{d}$ on the variation of displacements and stresses in the support. Some results are presented in the Fig.4:

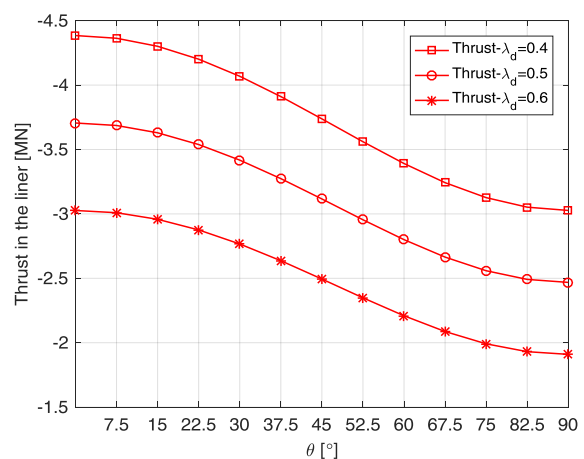

(a)

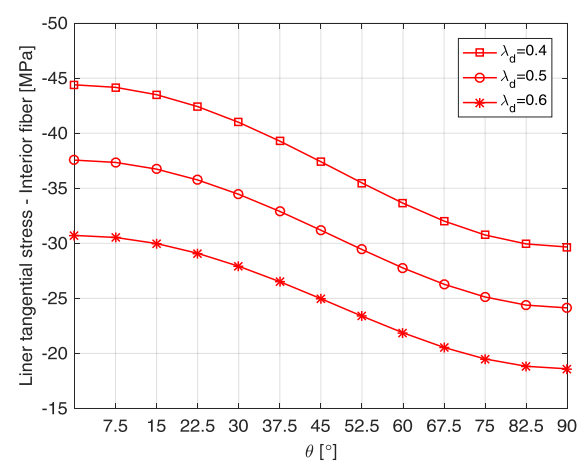

(c)

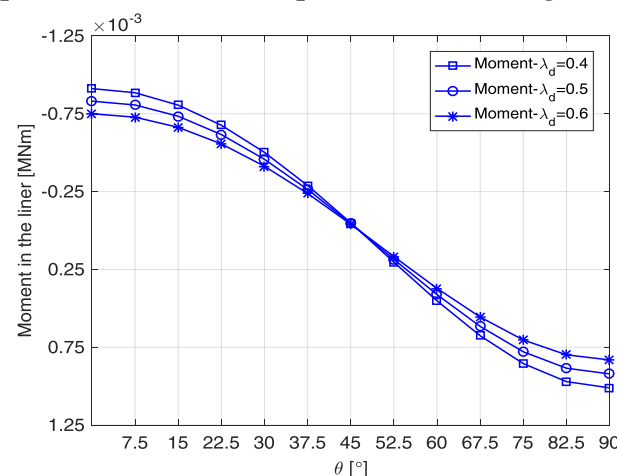

(b)

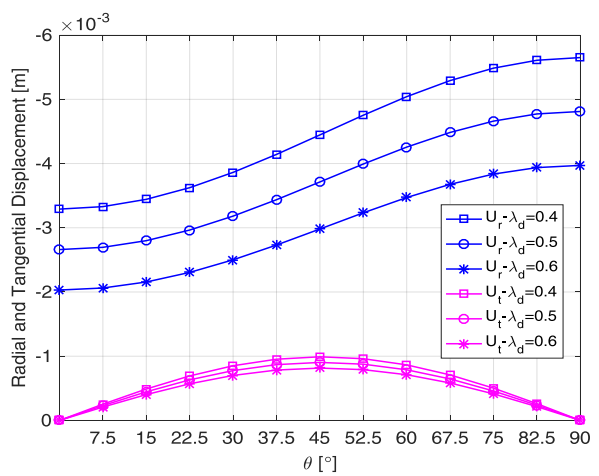

(d)

Fig.4. The thrust (a), moment (b), stresses (c) and the displacements (d) of the liner corresponding to $\lambda_{\mathrm{d}}=0.4, \lambda_{\mathrm{d}}=0.5$ and $\lambda_{\mathrm{d}}=0.6$ for the case of hydro-mechanical model. 
As a result, when the distance $\lambda_{d}$ increase, the thrust (Fig.4a) and thereby, the stresses as well as the displacements in the liner decrease (Fig.4c, d). These expected results explain that if the distance from the support installation section to the tunnel face is greater, the surrounding rock mass of the tunnel converged more significantly before placing the support. In other words, the internal forces are realised much more before support installation, and thus, the support is subjected to smaller pressures.

Nevertheless, it is known that the principle role of the liner/support is to limit the convergence of the tunnel wall as well as plastic deformation zone generated around the tunnel. Therefore, determining the appropriate instant (or the distance $d$ ) to install the support is an important issue, which ensures at once the bearing capacity of the support and limiting the displacement of tunnel wall as well as limiting the plastic deformation zone around the tunnel.

Fig. $4 \mathrm{c}$ shows also that, the liner stresses is always larger at the springline $\left(\theta=0^{\circ}\right)$ where the stiffness of the rock is greater. Thus, in tunnel design, the results calculated at this last point will be utilized in the design of support/liner.

\section{Conclusions}

In this work, a solution is built for an interactive problem between the support and the massif around the deep tunnel in the poroelastic anisotropic medium based on the approach of the classical convergence-confinement method. The solution could be considered as a quick and accurate analysis tool to calibrate preliminary the tunnel support/liner as well as the stress-strain state of the rock mass around the excavation for the tunnel design.

Some analysis pointed out that, the stresses and deformations of the support/liner are always greater in the case of considering the hydro-mechanical model. Some numerical results indicated also that, the greater stress of the support/liner occurs always in the larger stiffness direction, i.e., horizontal direction in this study. In addition, the distance from the support installation section to tunnel face influences strongly on the work of the support/liner in equilibrant state. Therefore, the calibration of the tunnel support of the tunnel in poroelastic anisotropic medium should be considered with the hydro-mechanical model and the stress state in larger stiffness direction as well as the deconfinement rate of support installation so that the support/liner meets at once requirements of bearing and limiting the convergence and the plastic deformation zone around the tunnel.

This research is funded by Vietnam National Foundation for Science and Technology Development (NAFOSTED) under grant number 107.01-2017.307.

\section{References}

1. Panet, M., Presses ENPC, (1995).

2. Einstein H.H., Schwartz C.W., J. Geotech. Engng. Div. ASCE, (1979)

3. Gill D.E., Leite M.H., Proc. 8th ISRM Cong., Tokyo, (1995)

4. Tran Manh, H. (Doctoral dissertation, Paris Est), (2014)

5. Tran, N. H., (Doctoral dissertation, Université d'Orléans), (2016).

6. Carranza-Torres, C., \& Fairhurst, C., Tunn Undergr Sp Tech, 15, 2 (2000).

7. Tran N. H., Do D. P. \& Hoxha D., Euro. Jour. Env. Civil Engin., (2017)

8. Flugge W., Stresses in Shells. Springer, New York, (1966)

9. Armand G., Noireta A., Zghondi J., Seyedi D.M.,. J. Rock Mech. Geotech. Eng, 5 (2013) 